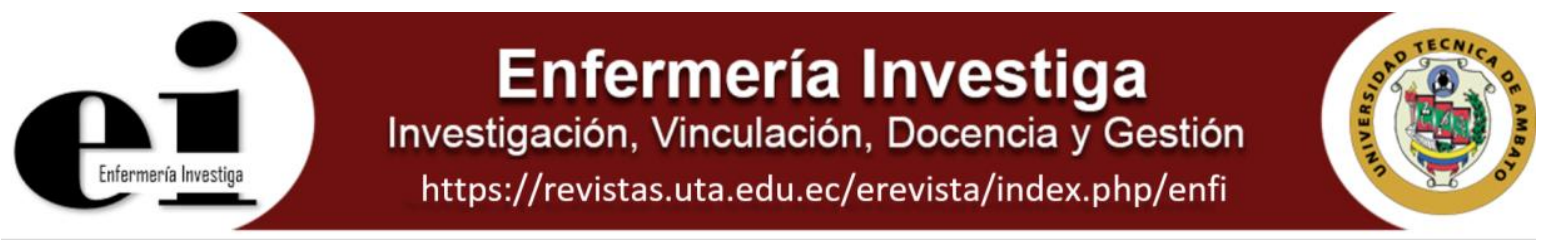

\title{
PARTO HUMANIZADO Y LA FUNCIÓN DE ENFERMERÍA EN LOS ESTABLECIMIENTOS DE SALUD PÚBLICA.
}

\author{
HUMANIZED DELIVERY AND THE NURSING ROLE IN PUBLIC HEALTH FACILITIES.
}

María de los Ángeles Cobo Pérez ${ }^{1}$ https://orcid.org/0000-0002-7646-2100, Eulalia Isabel Analuisa Jiménez ${ }^{2}$ https://orcid.org/0000-0002-7906-7640

${ }^{1}$ Estudiante de la Carrera de Enfermería, Universidad Técnica de Ambato, Ecuador.

${ }^{2}$ Docente de la Carrera de Enfermería, Universidad Técnica de Ambato, Ecuador

2477-9172 / 2550-6692 Derechos Reservados @ 2022 Universidad Técnica de Ambato, Carrera de Enfermería. Este es un artículo de acceso abierto distribuido bajo los términos de la Licencia Creative Commons, que permite uso ilimitado, distribución y reproducción en cualquier medio, siempre que la obra original es debidamente citada

Recibido: 17 de julio 2021

Aceptado: 30 de octubre 2021

\section{RESUMEN}

Introducción: la humanización del parto asegura el respeto de los derechos fundamentales, reproductivos y sexuales de la gestante y la familia, reduciendo complicaciones perinatales y morbi-mortalidad materno-infantil. Objetivo: Caracterizar el parto humanizado y las funciones de enfermería en los establecimientos de salud pública. Métodos: diseño documental con revisión bibliográfica sobre humanización de parto para lo cual se realizó una búsqueda en bases de datos como: PubMed, Taylor \& Francis, LILACS, MEDLINE, BVS, SCIELO, LATINDEX, GOOGLE ACADÉMICO, en idioma español e inglés. Resultados: En base a revisión y análisis de 40 artículos se pudo conocer que el parto humanizado es reconocido mundialmente como una estrategia eficiente para reducir complicaciones perinatales y lograr una maternidad satisfactoria y que a nivel latinoamericano; Brasil, Perú, Argentina, Ecuador, Venezuela y México ya cuentan con iniciativas del sector público, privado y de organizaciones civiles para promocionar parto humanizado, y con ello reducir las tasas de cesáreas, parto instrumentado, morbi-mortalidad materno infantil y lograr una maternidad satisfactoria, segura basada en prácticas no invasivas ni farmacológicas para el alivio del dolor, educación y autorización sobre procedimientos, autonomía, buen trato y respeto por sentimientos y creencias. Conclusiones: En el pato humanizado, el rol de enfermería es esencial sobre todo en los establecimientos públicos en donde se asume maltrato, negligencia o falta de respeto por el parto, por lo que la enfermera está llamada a educar a la parturienta sobre el trabajo de parto y parto.

Palabras clave: parto humanizado, atención perinatal, enfermería, recién nacido.

\section{ABSTRACT}

Introduction: the humanization of childbirth ensures respect for the fundamental, reproductive and sexual rights of the pregnant woman and the family, reducing perinatal complications and maternal and infant morbidity and mortality. Objective: To characterize humanized delivery and nursing functions in public health establishments. Methods: documentary design with bibliographic review on the humanization of childbirth for which a search was carried out in databases such as: PubMed, Taylor \& Francis, LILACS, MEDLINE, BVS, SCIELO, LATINDEX, GOOGLE ACADEMICO, in Spanish and English. Results: Based on a review and analysis of 40 articles, it was possible to know that humanized delivery is recognized worldwide as an efficient strategy to reduce perinatal complications and achieve a satisfactory maternity and that at the Latin American level; Brazil, Peru, Argentina, Ecuador, Venezuela and Mexico already have initiatives from the public and private sectors and civil organizations to promote humanized delivery, and thereby reduce the rates of cesarean sections, instrumented delivery, maternal and infant morbidity and mortality and achieve motherhood, satisfactory, safe based on non-invasive or pharmacological practices for pain relief, education and authorization on procedures, autonomy, good treatment and respect for feelings and beliefs. Conclusions: In the humanized duck, the role of nursing is essential especially in public establishments where abuse, neglect or lack of respect for childbirth is assumed, so the nurse is called to educate the woman in labor about the labor of labor and delivery.

Keywords: humanized delivery, perinatal care, nursing, newborn

Autor de correspondencia: Lic. Mg. Lic. Mg. Eulalia Isabel Analuisa Jiménez, correo electrónico: ei.analuisa@uta.edu.ec

\section{INTRODUCCIÓN}

La atención humanizada es la interacción entre los conocimientos de la ciencia y los valores, para establecer una asistencia de calidad centrada en la sensibilidad, en la reflexión sobre la práctica, la ciencia y el arte (1). La humanización de la salud tiene como objetivo central orientar la actuación en el proceso de atención de los pacientes, en su globalidad personal y tratando de ofrecer una asistencia integral, que responsa a las necesidades psicológicas, biológicas, sociales y espirituales, por esta razón humanizar es hacer digna a una persona, es decir coherente con los valores del ser humano (2).

El concepto de humanización a nivel del parto fue adoptado globalmente en Brasil con el Programa de Atención Prenatal, Parto y Nacimiento (PHPN, según sus siglas en portugués), creado en el año 2000 con el propósito de calificar la atención prenatal en cuanto a acceso y cobertura, pero también para mejorar los procesos de parto. Según el PHPN la humanización engloba una recepción digna de la tríada mujerneonato-familia, sobre la base de conductas éticas y solidarias, 
para lo cual la institución debe estar organizada con un ambiente acogedor, donde prevalezcan prácticas que rompan con el tradicional aislamiento de las gestantes, con la inclusión de procedimientos que contribuyan al adecuado seguimiento del trabajo de parto, dejando atrás conductas impersonales e intervencionistas que puedan poner en riesgo la salud de las mujeres y los recién nacidos $(3,4)$.

En la últimas décadas se ha podido observar un incremento considerable en la aplicación de varias prácticas de trabajo de parto que permiten iniciar, regular y terminar, este proceso fisiológico con el fin de mejorar los resultados tanto para las mujeres como para sus hijos, no obstante, estas prácticas en muchas ocasiones merman la capacidad de la mujer para dar a luz y afectan de manera negativa su experiencia de parto, lo que deja clara la necesidad de fomentar el parto humanizado (5).

A criterio de Cáceres y col., el parto de toda mujer debe tener un carácter humanista en el que prevalezca la calidad, el respeto de los derechos humanos y reproductivos de la gestante, pues en este periodo la mujer enfrenta miedo, dolor e incomodidad, factores que deben ser atendidos por el personal de salud y en especial por el de enfermería, para crear una experiencia agradable del parto en base a información, respeto, comprensión e individualización, de acuerdo a las singularidades y la autonomía de cada mujer, evitando intervenciones rutinarias e invasivas y mejorando e vínculo entre ella, el recién nacido la familia y el personal de salud porque solo así se reducirán las tasas de morbimortalidad obstétrica-neonatal (6). Desde este punto de vista el parto humanizado tiene una visión diferente del proceso de alumbramiento, porque se asume que el parto es el inicio y punto de partida de la vida y es por ello un proceso que afecta al resto de la existencia humana, de ahí que la humanización del parto constituye una necesidad urgente en el campo de la salud materno-infantil (7) y por estas razones el rol de enfermería debe tomar protagonismo para una asistencia de calidad con expresión humana durante el cuidado ofrecido, porque solo de esta manera se podrá alcanzar excelencia en la prestación de servicios y mejorar el desempeño profesional (8), partiendo de estos argumentos, la realización del presente estudio tiene como objetivo caracterizar el parto humanizado y las funciones de enfermería en los establecimientos de salud pública para resaltar el rol de enfermería en el trabajo de parto humanizado y evitar intervenciones innecesarias.

\section{MÉTODOS}

Diseño documental con revisión bibliográfica sobre humanización del parto, para lo cual se realizó una búsqueda en bases de datos como: PubMed, Taylor \& Francis, LILACS, MEDLINE, BVS, SCIELO, LATINDEX, GOOGLE ACADÉMICO, con el objetivo de recolectar la mayor cantidad de información sobre el tema de estudio. La búsqueda de información se realizó mediante los siguientes descriptores: parto humanizado, atención perinatal, enfermería, recién nacido, en idioma español e inglés.

Criterios de selección: se incluyeron revisiones sistemáticas, artículos científicos originales, revisiones e investigaciones científicas directamente relacionadas con el tema. Se excluyeron las revisiones o artículos que no correspondan a los últimos 5 años, que sean diferentes al idioma inglés y español y que no aportaron el cumplimiento del objetivo, el estudio se desarrolló con 40 fuentes bibliográficas.

\section{DESARROLLO}

De acuerdo a directrices de la Organización Mundial de la Salud, el parto debe ser una experiencia positiva de vida que cumpla o supere las creencias y expectativas personales y socioculturales previas de la mujer, lo que incluye el nacimiento en un ambiente seguro desde el aspecto clínico y psicológico, además de contar con el apoyo práctico y emocional continuo, es decir, acompañada en el momento del nacimiento y ser asistida por personal amable y con competencias técnicas adecuadas y tener protagonismo en las decisiones del proceso, aspectos que se alcanzan mediante la humanización del parto (5).

La humanización del parto es fundamental para garantizar la vida "porque para cambiar el mundo, primero hay que cambiar la forma de nacer" y para ello se requiere humanización, en este proceso el rol de Enfermería es clave pero en muchas ocasiones, los médicos o demás especialistas solo permiten la realización de procedimientos generales como cateterización venosa, administración medicamentosa, rasurado genital y la adopción de posturas ginecológicas, sin existir libertad profesional, para la evaluación del estado de la gestante, el apoyo emocional, el registro de signos vitales materno-fetales, la asistencia durante el alumbramiento, la revisión del canal vaginal, la realización de episiorrafía, los cuidados inmediato del recién nacido, el registro de datos e intervenciones concernientes al resultado del parto y recién nacido la historia clínica y agilizar procedimientos en casos de emergencia (1012).

En países como México, la funciones gineco-obstétricas de la Enfermera engloban: la valoración inicial de las condiciones maternas y fetales (signos vitales, maniobras de Leopold, valoración dinámica uterina, signos y síntomas del trabajo de parto, estabilización de la paciente), promover un estado de bienestar físico, mental y espiritual del binomio madre-hijo durante el parto (registro de signos vitales, contracciones, modificaciones cervicales, descenso fetal, hidratación), atención en periodo expulsivo (valoración de condiciones generales, posición, aseo, amniorresis, condiciones del periné, signos vitales, factores de riesgo y estabilización) y atención en alumbramiento (verificar signos vitales, alumbramiento espontáneo, integridad de placenta y membranas, involución uterina, uso de fármacos, revisión del conducto vaginal para establecer posibles complicaciones (13).

Pero estas acciones deben enmarcarse en el enfoque humanista no solo como una forma de garantizar una atención eficaz y respetuosa donde se evite el maltrato, negligencia, aplicación de procedimientos y tecnologías que lejos de garantizar seguridad ocasionan iatrogenia e instrumentación, sino como una forma de asegurar la salud materno-infantil (14), misma que se alcanza al promover el parto natural y reducir las tasas de cesáreas que no deben sobrepasar el 10\% a nivel mundial pero que en el contexto de Latinoamérica es un grave problema de salud pública al alcanzar un porcentaje global del $38 \%$; en Brasil es del 50\%, México del $43 \%$ y El Salvador con $25 \%$, y al exponer a la mujer a nuevos problemas de salud y un mayor riesgo de muerte (15) que en la región alcanza un aproximado de 9.000 mujeres por año, para lo cual varias naciones han desarrollado estrategias como el parto humanizado como una forma de reducir la mortalidad materna, estas acciones han dado buenos resultados, en países como Barbados donde se ha logrado una reducción del $56 \%$, Bolivia (61\%), Brasil (43\%), Ecuador (44\%), El Salvador (39\%), Guatemala (49\%), Haití (43\%), Honduras (61\%), Nicaragua (38\%), Perú (64\%) y República Dominicana (57\%) (16).

Pero pese a la importancia que tiene la humanización durante la labor y desenlace del parto, esta práctica no se encuentra estandarizada, por lo cual sus recomendaciones varían entre las distintas naciones: 
Así por ejemplo en Colombia, se ha reportado que el personal de salud aún está alejado de directrices de humanización asistencial, porque no se brinda acompañamiento continuo, existe ausencia de protocolos y guías de práctica clínica para el uso adecuado de y otros fármacos, no se permite la movilidad y adopción de posturas para el trabajo de parto y se siguen utilizando ampliamente procedimientos inadecuados como la amniotomía, episiotomía, acciones que traen como consecuencia oxitocina el quebrantamiento de la autonomía y dignidad de las mujeres gestantes y repercutiendo negativamente en la experiencia del parto (17). Además, se reconoce a la falta de asistencia humanizada en la mayoría de instituciones materno-infantiles como un grave problema de salud pública porque ocasiona experiencias negativas e insatisfacción debido a la escasez de apoyo familiar durante el evento, la falta de información y protagonismo materno generan conflictos entre la parturienta y el personal de salud lo que conlleva a partos más dolorosos, largos y ponen en evidencia la falta de atención humanizada $(18,19)$.

En cuba, los criterios de humanización del trabajo de parto aún están pendientes sobre todo en cuanto a: libre elección de la mujer respecto a la posición, libertad de movimiento, acompañamiento filial de personas seleccionadas, así como el manejo de prácticas intervencionistas que logren cambios de actitud en la mujer, donde el rol de los profesionales de salud es la piedra angular para mejores resultados perinatales favorables, sino también la valoración de la satisfacción de necesidades humanas (20).

En Perú, se reconocieron oportunamente las necesidades de mejorar el proceso del parto, pues en las parturientas se generó gran incidencia de estrés, tristeza, soledad, desamparo y problemas durante el trabajo de parto debido a ineficientes intervenciones y educación por parte del personal médico y de enfermería, lo que llegó a considerarse como violencia de los derechos fundamentales de la mujer (21). Sin embargo, a partir de 2015 se planteó en el marco legal la aplicación del parto humanizado contemplando dentro de sus normas el acompañamiento de la mujer y la elección de la posición del parto, lo que incrementó significativamente la satisfacción de la atención y el número de partos naturales (22).

En Brasil pese al reconocimiento normativo de la humanización del parto, aún hace falta intervenir porque los centros hospitalarios no han logrado brindar la libre elección del acto de dar a luz de manera natural, privada y familiar, no se respeta la elección de procedimientos, ni se da protagonismo a la madre (23), debido a que el personal de enfermería tiene limitaciones como: estructura física institucional, insuficiencia de alojamiento, trabajo en equipo ineficaz, recursos y materiales insuficientes, hacinamiento, profesionales con sobrecarga laboral y resistencia de la parturienta en la colaboración de ciertas situaciones con las cuales no se encuentra familiarizada (24).

Es por ello que en la actualidad se busca que prevalezca la autonomía, el respeto de los derechos de la mujer y la familia, el apoyo empático de los profesionales, el estímulo para el uso de métodos no invasivos y no farmacológicos para el alivio del dolor y otras molestias, la libertad de posición y palabra (25), donde el rol de enfermería se enfoca a facilitar las pautas para una respiración adecuada en cada paso de la labor de parto, favorece la libertad de circulación, el establecimiento del vínculo entre profesionales y mujeres en trabajo de parto, estimular la presencia de una pareja y el uso del contacto físico. Su desempeño en la enseñanza se guía en la conducción de cursos del proceso del parto en general, y también en la asistencia a la atención prenatal, el parto y atención posparto, atención ginecológica y salud neonatal (26). A más de ello, De Oliveira, $t$ al., expresan que la asistencia humanizada por parte del personal de Enfermería durante el trabajo de parto y el parto en Brasil, se centra en el alivio del dolor, físico y emocional, brindar comodidad, libertad de elección en la posición para el nacimiento de su bebé y el mejor método de parto, considerando las opiniones, preferencias y necesidades de la mujer, brindar el apoyo necesario (material, profesional y emocional) y ayudar a que la experiencia del proceso de parto de la mujer sea segura, tranquila, satisfactoria y feliz, además porque es fundamental tener en cuenta que brindar una atención humanizada a la mujer que vive el ciclo del embarazo puerperal también es un reflejo de calidad asistencial $(27,28)$.

En Argentina, las prácticas de parto humanizado van más allá de realizar un simple procedimiento, permiten reflexionar sobre las necesidades de las mujeres que se encuentran en labor de parto, mismas que deben ser atendidas con especial consideración por su estado de vulnerabilidad, en base a: oferta del máximo de informaciones y explicaciones sobre los procedimientos a realizar, libertad de posición y movimiento durante el trabajo de parto, utilización de técnicas no invasivas y no farmacológicas para el alivio del dolor durante el parto (masajes, técnicas de relajación), promoción del contacto precoz piel a piel entre el bebé y la madre, apoyo al inicio temprano de amamantamiento, valoración de las necesidades y expectativas de la madre en torno a la atención recibida y promoción del protagonismo materno ante cualquier circunstancia (29).

Bajo esta línea de argumentación, resulta conveniente traer a contexto a Muñoz y col., quienes mencionan que Chile es uno de los países que posee altas tasas de intervencionismo durante el parto y un escaso uso de métodos no farmacológicos para el alivio del dolor, situaciones que irrespetan las directrices propuestas mundialmente para humanizar el parto en el que debe promoverse el empoderamiento de la madre, existir acompañamiento de personas significativas, relaciones empáticas entre la parturienta y los profesionales de la salud y una mínima aplicación de intervenciones (30).

En Ecuador, la idea del parto humanizado, se basa en el respeto de los derechos humanos, reproductivos y sexuales de la mujer y sus parejas para lo cual se requiere una comunicación afectuosa entre padres y el equipo médico, atención personalizada con todos los conocimientos pertinentes, asistencia del equipo en acompañar a la pareja en el nacimiento de su bebé, respeto de miedos y angustias, evitar procesos innecesario y el respeto por formas culturales de alumbramiento como: posición vertical, ambiente cálido, uso de hierbas, baño caliente, entierro de placenta, corte del cordón y acompañamiento de esposo y otro familiar de género femenino (31). Además que, el parto humanizado debe permitir a la mujer practicar tradicional y libremente la posición del parto dentro de un ambiente seguro donde en caso de una emergencia obstétrica existan las condiciones necesarias para evitar secuelas maternas y neonatales (32).

En el estudio de Ortunio y col., se evidencia que tanto Ecuador como Venezuela han tenido una importante iniciativa desde el sector público y organizaciones civiles para promocionar parto humanizado, lo que constituyen claras expresiones de resistencia a la hegemonía biomédica lo que se ha logrado mediante la introducción del psicoprofiláctico y la formación de mujeres y profesionales para la atención comunitaria. Las prácticas de humanización incluyen el parto vertical, parto en agua, los saberes populares y desarticulando la atención estandarizada para que la mujer sea quien controle, decida y esté presente en todo momento (33).

En México, la atención del parto humanizado implica la atención de opiniones, necesidades y valoraciones 
emocionales de las mujeres y sus familias, para lo cual es necesario: reconocer a la madre e hijo como los verdaderos protagonistas, no interferir en el proceso a menos de un riesgo eminente, brindar atención personalizada y continua, favorecer la libertad de movimiento y posición de la mujer, promover una relación armónica, acompañamiento, privilegiar el vínculo inmediato entre madre-hijo y evitar procedimientos invasivos innecesarios (34).

Por lo expuesto, el parto humanizado es una modalidad de atención del parto caracterizada por el respeto a los derechos de los padres y los niños en el momento del nacimiento en el que se toma en consideración los valores de la mujer, sus creencias y sentimientos respetando su dignidad y autonomía para el empoderamiento de la mujer, para lo cual es necesario un trabajo interdisciplinario entre los actores: acompañante, parturienta, obstetra profesional y profesional médico pediátrico en el mejor de los casos (35).

Sin embargo en América latina donde las condiciones de salud son complejas por la poca accesibilidad a atención prenatal y obstétrica en algunas zonas y por rasgos culturalmente marcados de la medicina ancestral, sobresale como miembro importante del parto humanizado la figura de la partera tradicional o también denominada matrona, comadrona, partera empírica, misma que en países como Nicaragua, Paraguay, Argentina, Venezuela, Brasil, Panamá, Honduras, Uruguay y Costa Rica se consideran como parte de los programas de salud con roles de colaboradoras para el ejercicio del modelo biomédico en salud materna porque su práctica va más allá de asistir un procedimiento, sino que su sabiduría y accionar reconoce la importancia de traer una nueva vida al mundo en las mejores condiciones lo que implica acompañamiento, apoyo, uso de remedios caseros para el alivio del dolor y estrés, "acomodan al feto para el nacimiento", "fajan a la madre", "curan el ombligo", promueven el vínculo materno-fetal cercano, la lactancia temprana, lo que brinda a la madre mayor satisfacción del proceso de maternidad $(36,37)$.

Esta situación ha llevado a que actualmente se busquen mecanismos como la instrucción formal de la partera para garantizar partos seguros pero sin eliminar la naturaleza y esencia de la atención tradicional del parto, descrito por algunos autores como un acto dotado de sabiduría el cual difiere de la atención prestada en servicios modernos, donde se realiza en ambientes fríos, rígidos y técnicos que nada tiene que ver con la atención integral y humanizada que el traer una vida al mundo implica (38).

Finalmente, para Perkins y col., pese a que en las instituciones de maternidad se promueve la instauración del nacimiento humanizado que si bien ha logrado mejorar la atención, siguen existiendo barreras para lograr este objetivo entre ellas; las reglas y estrategias institucionales estrictas que restringen el acompañamiento a la mujer en labor de parto y restricción en la elección de procedimientos (39), situación que han empeorado actualmente con la pandemia COVID-19 porque debido a protocolos de bioseguridad se ha optado por protocolos rígidos de atención obstétrica, practica de cesáreas, atención instrumentada y mecanizada, irrupción del vínculo madre-bebé y lactancia, lo que va en contra del parto humanizado (40).

\section{CONCLUSIONES}

REFERENCIAS
La revisión bibliográfica ha puesto al descubierto que el uso de ciertos procedimientos médicos, lejos de facilitar y garantizar seguridad en el proceso del parto, ocasionan iatrogenia, aumentando la proporción de cesáreas e instrumentación y la obstaculización del vínculo madre e hijo, razón por la cual es necesaria la adopción del enfoque humanizado en el que debe prevalecer el trato digno y respetuoso, autonomía corporal, empoderamiento durante la atención, acompañamiento, reducción o eliminación de intervenciones médicas y farmacológicas innecesarias que son frecuentes en países como Colombia, Chile, Cuba, donde existen altas tasas de intervencionismo en el parto, escaso uso de métodos no farmacológicos y limitaciones en la participación, movilidad y acompañamiento de la parturienta, situaciones que irrespetan las directrices propuestas mundialmente para humanizar el parto.

La humanización del parto debe ser entendido como un proceso de atención integral más allá de los protocolos y la evidencia científica gíneco-obstetra, maternidad o paternidad responsable, sino como un conjunto de elementos necesarios para lograr que el proceso del parto constituya un acontecimiento trascendental en la vida de la mujer, donde se develan sensaciones, percepciones y sentimientos mediados por la autorrealización personal y satisfacción de las necesidades humanas, aspectos que han sido reconocidos por varios países a nivel mundial y latinoamericano, entre ellos Brasil, Perú, Argentina, Ecuador, Venezuela, México donde existen iniciativas del sector público, privado y de organizaciones civiles para promocionar el parto humanizado y con ello reducir las tasas de cesáreas, parto instrumentado, morbi-mortalidad materno infantil y lograr una maternidad satisfactoria, segura basada en prácticas como el acompañamiento, libertad de posición para el alumbramiento (parto vertical), utilización de técnicas no invasivas ni farmacológicas para el alivio del dolor, educación y autorización sobre procedimientos, autonomía, buen trato y respeto por sentimientos y creencias.

En el enfoque del pato humanizado, el rol de enfermería es esencial sobre todo en los establecimientos públicos en donde se asume maltrato, negligencia o falta de respeto por el parto lo que puede constituirse como una violación de los derechos humanos y reproductivos por lo que la enfermera está llamada a educar a la parturienta sobre el trabajo de parto y parto, así como a brindar explicación de los procedimientos a realizarse, asistir con técnicas de relajación para el alivio del dolor, favorecer la libertad de circulación, estimular la presencia de un acompañante, buscar la mejor posición para el alumbramiento, verificar el estado materno-infantil, promover el trabajo interdisciplinario y agilizar procedimientos en casos de complicaciones.

\section{CONFLICTO DE INTERESES \\ Ninguno declarado}

\section{FINANCIACIÓN}

Aufinanciado

\section{AGRADECIMIENTOS}

Ninguno 
Llanes G, Bejarano D, Márquez L, Ponce C, Martínez R. La humanización de la atención de enfermería en salud laboral. Revista Enfermería del Trabajo.2018;8(1):18-26. Disponible en: https://dialnet.unirioja.es/servlet/articulo?codigo=6536488

Correa M. La humanización de la atención en los servicios de salud: un asunto de cuidado. Revista Cuidarte.2017;7(1):1227-1231. Disponible https://revistacuidarte.udes.edu.co/index.php/cuidarte/article/view/300/527

Batista A, Alende L, Cremonese L, Scarton J, Neumaier C, Ressel L. Humanization of childbirth: meanings and perceptions of nurses. Escola Anna Nery. 2017; 21(4): 1227-1231. Disponible en: https://doi.org/10.1590/21779465-ean-2016-0366

Cáceres F, Nieves G, Lizarazo A. Elaboración y validación de una cartilla sobre parto humanizado. Enfermería Global.2020;19 (60):64-72. Disponible en: https://revistas.um.es/eglobal/article/view/412771/287141

Organización Mundial de la salud. Recomendaciones de la OMS para los cuidados durante el parto, para una experiencia de parto positiva. 2018 [citado 2020 Noviembre 10. Disponible en: https://apps.who.int/iris/bitstream/handle/10665/272435/WHO-RHR-18.12-spa.pdf?ua=1.

Cáceres F, Nieves G. Atención humanizada del parto. Diferencial según condición clínica y social de la materna. Revista Colombiana de Obstetricia y Ginecología. 2017; 68(2): 128-134. Disponible en: http://www.scielo.org.co/pdf/rcog/v68n2/v68n2a05.pdf

Madeiros R, Teixeira R, Nicolini A, Alvares A, Correa A.Humanized Care: insertion of obstetric nurses in a teaching hospital. Semantics. 2016;69(6):1029-36. Disponible en: https://www.scielo.br/pdf/reben/v69n6/en_0034-7167reben-69-06-1091.pdf

Borges L, Pérez A, Sánchez R. Influencia del desempeño de los profesionales de enfermería en la atención humanizada al parto. Revista cubana de Enfermería. 2018; 34(2). Disponible en: http://revenfermeria.sld.cu/index.php/enf/article/view/1426/357

Guirao G, Silamani J. Utilidad y tipos de revisión de lieratura. Revista de Enfermería ENE. 2015; 9(2). Disponible en: http://ene-enfermeria.org/ojs/index.php/ENE/article/view/495/guirao

Tavera R, Aveiga M, Gómez A. Papel de la enfermera como doula en la labor de parto humanizado. Revista Dilemas Contemporáneos: Educación, Política y Valores. 2019;7(125): 1-12 Disponible en: https://www.dilemascontemporaneoseducacionpoliticayvalores.com/index.php/dilemas/article/view/1019/148

Lafauries M, Angarita M, Chilatra C. Atención humanizada del embarazo: la mirada de gestantes que acuden a una unidad hospitalaria de salud. Enfermería Actual de Costa Rica. 2020;(38): 1-16. Disponible en: https://www.scielo.sa.cr/pdf/enfermeria/n38/1409-4568-enfermeria-38-180.pdf

Ministerio de salud El Salvador. Guías de atención de enfermería en ginecoobstetricia y perinatología. San Salvador: Dirección de Regulación y Legislación en Salud. 2019: 1-169. Disponible en: http://asp.salud.gob.sv/regulacion/pdf/guia/guias_de_atencion_de_enfermeria_en_ginecoobstetricia_y_perinatolo gia_marzo2019.pdf

Secretaría de Salud. Modelo de atención de Enfermería obstétrica. Enfermería obstétrica. 2016. Disponible en: http://www.cpe.salud.gob.mx/site3/publicaciones/docs/modelo atencion obstetrica.pdf

Downe S, Finlayson K, Oladapo O, Bonet M, Gulmezoglu A. What matters to women during childbirth: A systematic qualitative review. Plos One. 2018;13(4): e0194906. Disponible en: https://www.ncbi.nlm.nih.gov/pmc/articles/PMC5903648/

Organización Panamericana de la Salud. Las cesáreas. 2016 [citado 2020 Noviembe 05. Disponible en: https://www.paho.org/nic/index.php?option=com_docman\&view=download\&category_slug=datos-yestadisticas\&alias=696-boletin-informativo-sobre-cesareas\&ltemid=235.

Organización Panamericana de la Salud. Once países de América Latina y el Caribe registraron avances en la reducción de la mortalidad materna, según nuevo informe de la ONU. 2016 [citado 2020 Noviembre5. Disponible en: https://www.paho.org/uru/index.php?option=com_content\&view=article\&id=839:once-paises-de-america-latinay-el-caribe-registraron-avances-en-la-reduccion-de-la-mortalidad-materna-segun-nuevo-informe-de-laonu\&ltemid= 451 .

Jiménez G, Peña Y. Adherencia a las recomendaciones de la OMS en la atención del parto y nacimiento humanizado. Medellín, Colombia. Revista de la Universidad Industrial de Santander. 2018; 50(4):320-327. Disponible en: http://www.scielo.org.co/scielo.php?script=sci_arttext\&pid=S0121-08072018000400320

Giraldo D, González E, Henao C. Experiencias de las mujeres durante el trabajo de parto y parto. Revista Avances de enfermería.2015;33(2):271-281. Disponible en: http://www.scielo.org.co/pdf/aven/v33n2/v33n2a09.pdf

Beltrán O. Impersonal Care or Humanized Care: a Decision Made by Nurses? Hourglass Model. Investigación y $\begin{array}{lll}\text { Educación } & \text { en } & \text { Enfermería. }\end{array}$

Borges L, Sánchez R, Dompinguez R, Sixto A. El parto humanizado como necesidad para la atención integral a la mujer. Revista cubana de Obstetricia y ginecología. 2018;44(3). Disponible en: http://revginecobstetricia.sld.cu/index.php/gin/article/view/226/293

Centenaro D, Marin A, Da Silva C, Golembiewski M. ¡Llegó la hora! Expectativas y experiencias de parto de adolescentes embarazadas. Revista de Psicología. 2015;33(2):1-29. Disponible en: http://www.scielo.org.pe/pdf/psico/v33n2/a04v33n2.pdf 
22.

23.

24.

25.

26.

27.

28.

29.

30.

31.

32.

33.

34.

35.

36.

37.

38.

39.

40.

González E. Parto humanizado: percepción del acompañante en un Instituto Especializado de Lima. Revista Internacional de salud materno fetal. 2018;3(4):17-22. Disponible en: http://ojs.revistamaternofetal.com/index.php/RISMF/article/view/80/88

Da Silva I, Santos S, Teles M, Cipriano V, Melo T. Intervención educacional para la mejora en la asistencia al trabajo de parto normal. Enfermería Global. 2020;19(2): 226-256. Disponible en: https://dialnet.unirioja.es/servlet/articulo?codigo=7328531

Lessa E, Da Silva T, Rodrígues L, Fragoso A, Teixeira R, DE Oliveira M. The humanization in the assistance to delivery and childbirth. Revista de Enfermagen. 2018; 12(8): 2154-2162. Disponible en: https://periodicos.ufpe.br/revistas/revistaenfermagem/article/view/236334/29730

Santos P, Menezes T, Pereira N, Moura L, Rodrígues K, Eichenberg F. Best practices in the delivery process: conceptions from nurse midwives. Revista Brasileira de Enfermagem. 2019; 72(2):455-462. Disponible en: https://www.scielo.br/pdf/reben/v72n2/0034-7167-reben-72-02-0455.pdf

Furtado H, Makuch M. Nursing Training and Practice on Humanization Actions in Monitoring the Delivery in Brazil. International Medical Society. 2016;9(212):1-12. Disponible en: http://imed.pub/ojs/index.php/iam/article/view/1792/1360

Vieira M, Santos A, Silva J, Sánches M. Obstetrical nursing care based on good practices: from admission to delivery. Revista electrónica de Enfermería. 2016;18: E1166. Disponible en: https://docs.bvsalud.org/biblioref/2017/04/832816/36714-182176-1-pb.pdf

Cavalcante M, Coutinho L, Ferraz E, Oliveira L, Silva S. Perceptions of nursing professionals about humanization of childbirth in a hospital environment. Revista Rene. 2019; 20: e41409. Disponible en: http://periodicos.ufc.br/rene/article/view/41409/99341

. Contreras M, Guaymas M. Cuidados de Enfermería para un trabajo de parto humanizado. Notas de Enfermería. 2017; 17(29): 9-15. Disponible en: https://pesquisa.bvsalud.org/portal/resource/pt/biblio-869158?lang=es

Muñoz C, Contreras Y, Manríquez C. Vivencias de mujeres con asistencia de parto personalizado. Revista chilena de obstetricia y ginecología. 2018;83(6):586-594. Disponible en: https://scielo.conicyt.cl/pdf/rchog/v83n6/07177526-rchog-83-06-0586.pdf

Macías M, Haro J, Piloso G, Galarza M, Quishpe B.. Importancia y beneficios del parto humanizado. Revista
$\begin{array}{llll}\text { Dominio } & \text { en } & \text { Ciencias. } & \text { 2018;4(3):392-415. }\end{array}$ https://dominiodelasciencias.com/ojs/index.php/es/article/view/815

Macías M, Tocoamán I, Giler L, Quimis W, Choez M, Álvarez M. Parto humanizado como estrategia de salud pública en atención primaria de salud. Revista Científica Mundo de la Investigación y el Conocimiento. 2018;2(3):730-745. Disponible en: https://dialnet.unirioja.es/servlet/articulo?codigo=6732823

Ortunio E, Khan T. Estrategias del parto y nacimiento humanizado en la promoción de la maternidad segura en países de la Alianza Bolivariana en América. Comunidad y Salud. 2019; 17(2):46-61. Disponible en: https://pesquisa.bvsalud.org/portal/resource/pt/biblio-1127836

Calleja A, Contreras C, Quintero F, Mainero G. Nacimiento humanizado: aportes de la atención intercultural a las mujeres en el embarazo, parto y puerperio. Género y salud. 2016;10(2): 1-16. Disponible en: https://www.corteidh.or.cr/tablas/r37545.pdf

Fonseca L, Monteiro J, Aredes N, Bueno J, Domíngues A, Coutinho V. Escenario de simulación interdisciplinaria en educación en enfermería: parto y nacimiento humanizados. Revista Latino-Americana de Enfermagem. 2020; 28: e3286. Disponible en: https://www.scielo.br/pdf/rlae/v28/0104-1169-rlae-28-e3286.pdf

García A, Navarro C, Pabón Y, Brito K, Freyle Y. La partería tradicional. Revista Cubana de Enfermería. 2018; 34(2): 384-393. Disponible en: http://scielo.sld.cu/pdf/enf/v34n2/1561-2961-enf-34-02-e1657.pdf

Carvajal R, Gomez M, Restrepo N, Varela M, Navarro M, Angulo E. Panorama académico y político que enfrentan las parteras tradicionales en América Latina. Revista Cubana de Salud Pública. 2018; 44(3): e1061. Disponible en: http://scielo.sld.cu/pdf/rcsp/v44n3/1561-3127-rcsp-44-03-e1061.pdf

8. Laureano E, Villaseñor J, Farías M, Mejía M, Ramírez H. Ejercicio tradicional de la partería frente a su profesionalización: estudio de caso en Jalisco, México. Revista Facultad Nacional de Salud Pública. 2016; 34(3): 275-283. Disponible en: https://www.redalyc.org/pdf/120/12046949002.pdf

Perkins J, Ehsanur A, Mhajabin S, Bakkar A, Mazumder T, Rifat M. Humanised childbirth: the status of emotional support of women in rural Bangladesh. Sexual and Reproductive Health Matters. 2019;(9): 228-247. Disponible en: https://www.tandfonline.com/doi/full/10.1080/26410397.2019.1610277

Alves V, Caroci A, Pererira K, Girardi N, Caroi A, González M. Recomendaciones asistenciales a la mujer en el parto y postparto y al recién nacido durante la pandemia de COVID-19: revisión sistemática exploratoria. Revista Latino-Americana de Enfermagem. 2020;28:e3359. Disponible en: https://www.scielo.br/pdf/rlae/v28/0104-1169rlae-28-e3359.pdf 